\title{
Neuropilin 1 in uterine leiomyosarcoma. Clinical and pathological analysis
}

\author{
Marcin Bobiński ${ }^{1}$, Karolina Okła ${ }^{1}$, Jan Kotarski ${ }^{1}$, Justyna Szumiło ${ }^{2}$, Grzegorz Polak ${ }^{1}$, \\ Małgorzata Sobstyl' ${ }^{1}$, Wiesława Bednarek ${ }^{1}$ \\ ${ }^{1} 7^{\text {st }}$ Chair and Department Of Gynaecological Oncology and Gynaecology Medical University in Lublin, Poland \\ ${ }^{2}$ Chair and Department of Clinical Pathomorphology, Medical University in Lublin, Poland
}

\begin{abstract}
Objectives: The role of angiogenesis in leiomyosarcomas still remains unclear. The aim of this study was to evaluate the NRP1 expression in the leiomyosarcoma tissues and to find the relations between its expression and the clinical features.

Material and methods: The study group consisted of 50 patients with diagnosis of the uterine leiomyosarcoma. Clinical and follow up data were collected. Using immunohistochemical methods the expression of NRP1 was detected.

Results: The lack of NRP1 expression was found in 14 cases, positive (weak or moderate) expression was noted in 36 cases. The significantly higher expression of NRP1 was observed in more severe clinical stages in comparison to lower stages of the disease. The significantly shorter survival of patients with the positive expression of NRP1 in leiomyosarcoma was observed.

Conclusions: The expression of NRP1 is associated with clinical advancement and worse prognosis in uterine LMS. Neuropilin 1 can be widely used as a postoperative survival predictor for the patients suffering from uterine LMS.
\end{abstract}

Key words: uterine sarcoma, neuropilin 1, angiogenesis

\section{INTRODUCTION}

Uterine leiomyosarcomas (LMS) constitute a rare type of female reproductive organs tumors. It is a heterogeneous group of cancers whose common feature is the histological origin from the mesenchymal tissues. These tumors are characterized by the dynamic development, the capacity to metastasize and postoperative recurrence [1]. The most common histological type of uterine sarcoma is leiomyosarcoma which is diagnosed in 1 to $8.4 \%$ of malignant uterine tumors $[2,3]$.

The cells with the high dynamics of the metabolic processes, such as cancer cells require permanent supplies in substrates and the discharge of metabolic products for their development, and most of all, for their mitotic activity. The process of angiogenesis often occurs simultaneously with the formation of lymphatic vessels [4]. The process of tumor angiogenesis has been the subject of many studies, meanwhile the processes of lymphatic vessels growth, formation of lymph nodes metastases and lymphangiogenesis induced by the tumor are still much less understood. Both lymphatic and blood vessels seem to play a crucial role in maintaining homeostasis in cancer cells. As is widely known, many cancers metastasize to lymphatic nodes predominantly so the development of the cancerous lymphatic vessels seems to be crucial for their spreading. The invasion of the lymphatic nodes constitutes a prognostic factor in some types of cancer such as melanoma, breast and prostate cancer [5]. VEGF family of proteins, especially VEGF-C and VEGF-D and their binding to VEGFR-3 $[6,7]$ play crucial role in the process of lymphangiogenesis. The recent research results indicate also a vital role of neuropilin family in the regulation of this process.

Neuropilins are a group of transmembrane proteins with a molecular weight of approx. $130 \mathrm{kDa}$. They serve as receptors for many important factors regulating cell metabolism. These are VEGF proteins, class 3 semaphorins, PIGF-2, FGF, HGF, PDGF, TGF- $\beta$ [8]. Physiologically, neuropilins appear on the surface of many types of cells such as endothelium 
of blood and lymphatic vessels, T lymphocytes, nerve cells, keratinocytes. Neuropilins are expressed in certain types of cancers, of both epithelial and mesenchymal origins (e.g.: certain leukemias, malignant melanoma, pancreatic cancer, osteosarcoma, tumors of the gastrointestinal tract) $[9,10]$. The presence of the NRP has been demonstrated in many other malignant tumors, including lung cancer or malignant gliomas [11-13]. Neuropilin family consists of two proteins encoded by two separate genes NRP 1 (located in 10p12) and NRP 2 (located in 2q34) [14]. In spite of the structural similarities, both neuropilins have an affinity for different ligands. Neuropilin 1 binds to VEGF-A, PIGF2, SEMA3A, SEMA3B semaphorins and SEMA3E. On the other hand, neropilin 2 indicates greater affinity to VEGF-C, VEGF-D, SEMA3F and SEMA3G semaphorins [8]. It should be noted that different ligands which bind to neuropilins compete with each other. This information can be used when planning future targeted cancer therapies due to the fact that neuropilins combined with different ligands can activate various cell pathways. An example of such relation is the competitive binding of neuropilin 1 with VEGF and class 3 semaphorin. In the first case, with the contribution of VEGFR-2, the endothelial cells migration is stimulated. In the second case, neuropilin 1 together with plaxin A causes the opposite reaction [15].

Neuropilins play also an important role in the angiogenesis and lymphangiogenesis processes. Neuropilin 1 shows a co-receptor activity for VEGFR-2 facilitating its binding to VEGF (VEGF A), on the other hand, neuropilin 2 performs this function for VEGFR-3 and its ligands VEGF-C i VEGF-D [16]. What seems to be interesting is the influence of the proteins from the VEGF family onto mutual neuropilins location and VEGF receptors. It has been shown that the exposure on the endothelial growth factors influences the neuropilin location in the vicinity of VEGF receptors [17]. During lymphangiogenesis neuropilin 1 is involved in the formation of lymphatic vessel valves as a receptor for class 3 semaphorins The expression of neuropilin 2 is higher in endothelial cells and together with VEGF-C ligand it influences budding and growth of lymphatic vessels [18].

\section{OBJECTIVES}

The aim of this study was to evaluate the neuropilin 1 expression in the leiomyosarcoma tissues and to find the relations between its expression and the clinical stage of the disease and the overall survival of patients .

\section{MATERIAL AND METHODS}

The study group consisted of 50 patients with the uterine leiomyosarcoma diagnosis, operated in the $1^{\text {st }}$ Chair and Department Of Gynaecological Oncology and Gynaecology Medical University in Lublin, in 2000-2013. The average age of patients was 52.84 years old (median 51.50, SD 12.36, min. 29, max. 76). The ethnicity of all of the patients in study group were white, non hispanic, non latino. The patients were enrolled to the study retrospectively. In each case, the degree of clinical stage was evaluated according to FIGO classification effective from 2009 on the basis of surgeries descriptions and histopathological examination [19].

46 (92\%) of patients underwent total hysterectomy and bilateral salpingoopherectomy. In two cases (4\%) the surgery was limited to tissue sampling, 1 patient (2\%) underwent total hysterectomy without adnexectomy, in another case (2\%) retroperitoneal tumor was resected.

\section{Immunohistochemistry}

From the paraffin blocks containing representative pieces of tissue taken from leiomyosarcoma the microscopic preparations were made. The immunohistochemical examinations were made on the scraps measuring $4 \mu \mathrm{m}$. After dewaxing and hydration of the tissue material in the alcohol series, the procedure of unveiling antigenic determinant was applied by using EnVision FLEX Target Retrieval Solution High pH (50x) buffer, (Dako, Cat. No. K8000) for 40 minutes. Endogenous peroxidase activity was blocked with $3 \%$ sodium dihydrogen dioxide for 5 minutes at room temperature. The preparations were washed every time $3 \times 5$ min in EnVision Flex Wash Buffer (20x) (DAKO , Cat. No. K800721) and incubated with the primary antibody directed against an examined antigen NRP 1. In the studies, monoclonal mouse antibodies targeted against human protein neuropilin1 were used (Santa Cruz Biotechnology, USA) and a stain set DAKO EnVision+ System Kit with the secondary antibody conjugated with peroxidase. The color reaction was achieved after approx. 3 minutes of incubation at room temperature. Next, the cells nuclei were stained with the use of Mayer's hematoxylin. When performing a negative control, a procedure similar to that in the primary reaction was applied yet without the primary antibody. The reactions were evaluated with the use of light microscope Carl Zeiss Axiostar plus (Germany).

To evaluate the immunohistochemical reaction for neuropilin 1, a qualitative scale was adopted, based on the percentage of tumor cells exhibiting a color reaction [20],

- 0 - lack of cytoplasmic color reaction in cells,

- 1 - weak cytoplasmic staining in $\leq 10 \%$ of cells,

- 2 - moderate cytoplasmic staining in $>10-50 \%$ of cells,

- 3 - strong cytoplasmic staining in $>50 \%$ of cells.

\section{The statistical analysis}

The statistical analyses were prepared with the use of SPSS program, 14PL version. Qualitative variables (neuropilin 1 expression) were evaluated by the $\mathrm{Chi}^{2}$ and Fisher's tests. The correlations among variables were evaluated by the Spearman's test. The distribution of survival times were analyzed using the Kaplan-Meier method. The differences 
of survival times distributions in the studied groups were analyzed by the log-rank test (Mantel-Cox). The differences or relations between variables were considered as statistically significant at $\mathrm{p}<0.05$.

\section{RESULTS}

The lack of neuropilin 1 expression was found in $14 \mathrm{ca}-$ ses (28\%) of leiomyosarcoma, positive (weak or moderate) expression was noted in 36 cases (72\%). There was no strong neuropilin expression found. The examples of typical immunohistochemical staining results of the representative samples of leiomyosarcoma are presented in Figures 1-3.

For the statistical aims, the neuropilin 1 expression was recognized as qualitative variable and analyzed in two ways:

- analysis 1:0 - no expression vs 1 - expression i.e. from 1 to 2 (weak and moderate),

- analysis 2: 1 - no expression or weak expression i.e. from 0 to 1 vs 2 - moderate expression.

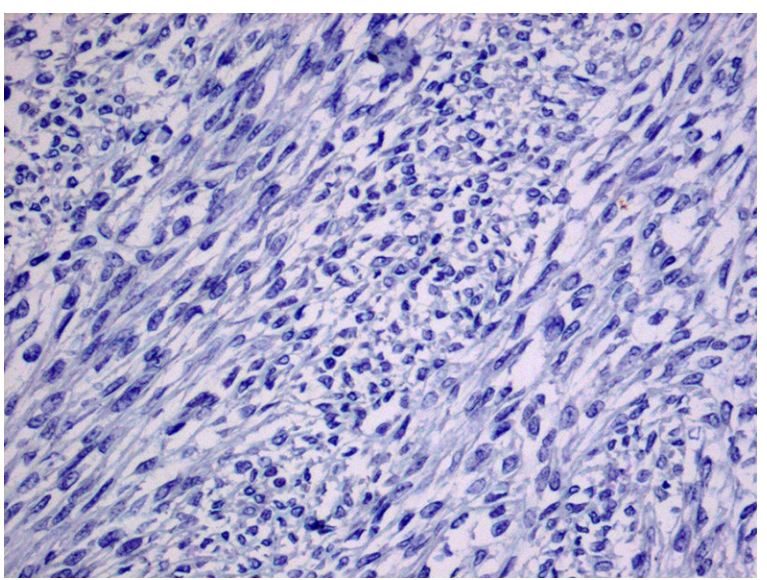

Figure 1. Negative immunohistochemical reaction to neuropilin 1 in leiomyosarcoma cells (Dako EnVision+ System-HRP; 20x lens magnification)

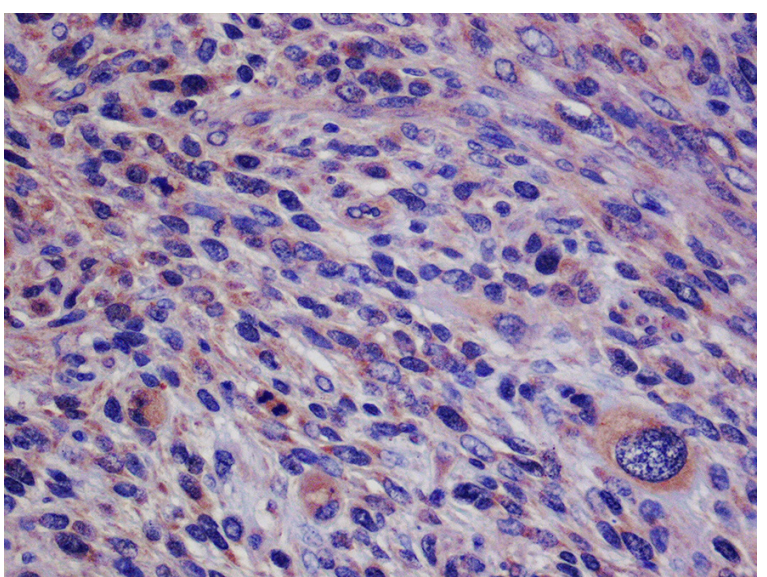

Figure 2. Weak positive cytoplasmic immunohistochemical reaction to neuropilin 1 in leiomyosarcoma cells (Dako EnVision+ System-HRP; $20 x$ lens magnification)

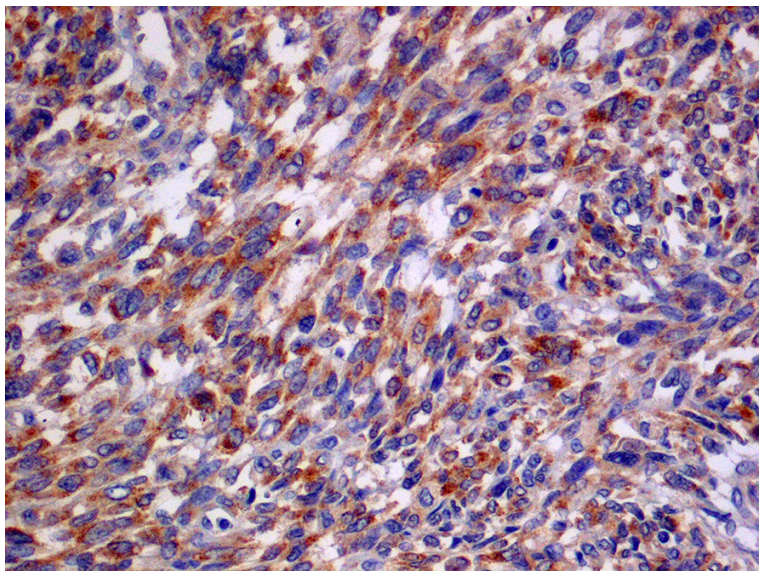

Figure 3. Moderately intensive positive cytoplasmatic immunohistochemical reaction to neuropilin 1 in leiomyosarcoma cells (Dako EnVision+ System-HRP; 20x lens magnification)

The positive correlation was found between intensity of neuropilin 1 expression (no expression vs expression) and stage of disease $(p=0.092)$. There were no significant differences $(p=0.111)$ in neuropilin 1 expression (no expression vs expression) depending on the degree of the disease stage considering the FIGO I + II and FIGO III + IV classification analyzed together. The significant differences $(p=0.015)$ in neuropilin 1 expression were noted (no expression or weak expression vs moderate expression) depending on the degree of the disease stage analyzed separately. The significantly higher $(p=0.002)$ expression of neuropilin 1 was observed in more severe clinical stages of leiomyosarcoma (FIGO III and IV) in comparison to lower degrees of disease stages (FIGO I and II) (Tab. 1 and 2).

While evaluating the relations between neuropilin 1 expression and an overall survival of patients, the significantly shorter $(p=0.018)$ survival of patients with the positive expression of neuropilin 1 in leiomyosarcoma was observed (Fig. 4). Significantly shorter $(p=0.006)$ survival of patients with the moderate expression of neuropilin 1 was demonstrated in comparison to patients with a lack of expression or low expression of this protein in leiomyosarcoma. The significantly higher neuropilin 1 expression was observed among patients of less than or equal to 2 years of survival, in comparison with patients with the survival rate more than 2 years. The significantly higher $(p=0.011)$ neuropilin 1 expression was noted in the group of patients with the survival rate less than or equal 2 years, in comparison with patients of the survival rate longer than 2 years (Tab. 3).

\section{DISCUSSION}

Among malignant cancers of female reproductive organs, LMS is one of those which appears rarely. In spite of the unquestionable progress which has been made in oncology for the past few years, both diagnostic process 
Table 1. Neuropilin 1 expression depending on the disease stage according to FIGO (IA-IV)

\begin{tabular}{|l|c|c|c|c|}
\hline & N & No expression & Expression & p* $^{*}$ \\
\hline FIGO IA & 10 & $6(60.0 \%)$ & $4(40.0 \%)$ & 0.092 \\
\hline FIGO IB & 23 & $6(26.1 \%)$ & $17(73.9 \%)$ & \\
\hline FIGO II & 6 & $1(16.7 \%)$ & $5(83.3 \%)$ & $4(80.0 \%)$ \\
\hline FIGO III & 5 & $1(20.0 \%)$ & $6(100.0 \%)$ & 0.111 \\
\hline FIGO IV & 6 & $0(0.0 \%)$ & $26(66.7 \%)$ & $10(90.9 \%)$ \\
\hline
\end{tabular}

$\mathrm{P}^{*}$ - the significance was calculated with the use of $\mathrm{Chi}^{2}$ test

Table 2. Neuropilin 1 expression (no expression or weak expression vs moderate expression) depending on disease stage according to FIGO classification

\begin{tabular}{|l|c|c|c|c|}
\hline & N & $\begin{array}{r}\text { No expression or weak } \\
\text { expression }\end{array}$ & Moderate expression & p $^{*}$ \\
\hline FIGO IA & 10 & $8(80.0 \%)$ & $2(20.0 \%)$ & 0.015 \\
\hline FIGO IB & 23 & $13(56.5 \%)$ & $10(43.5 \%)$ & $2(33.3 \%)$ \\
\hline FIGO II & 6 & $4(66.7 \%)$ & $4(80.0 \%)$ & 0.002 \\
\hline FIGO III & 5 & $1(20.0 \%)$ & $6(100.0 \%)$ & $14(35.9 \%)$ \\
\hline FIGO IV & 6 & $0(0.0 \%)$ & $10(90.9 \%)$ & \\
\hline
\end{tabular}

$\mathrm{P}^{*}$ - the significance was calculated with the use of $\mathrm{Chi}^{2}$ test

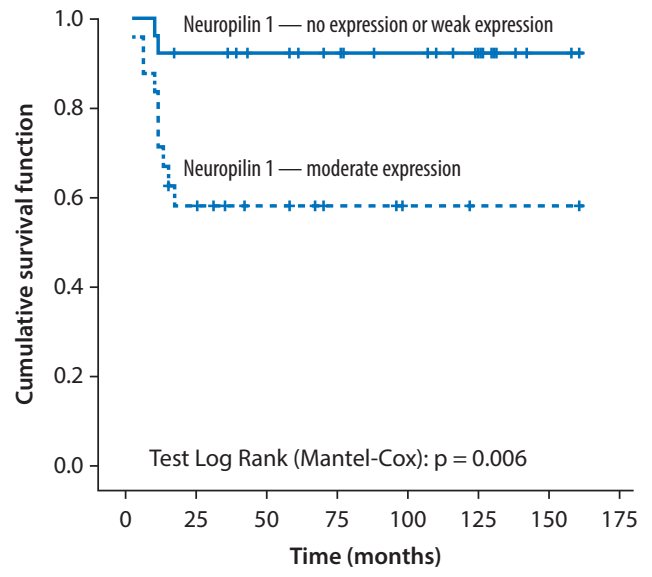

Figure 4. The cumulative survival function according to KaplanMeier for patients with leiomyosarcoma depending on neuropilin 1 expression. The trimmed mean (+), (no expression + weak expression vs moderate expression)

and the therapy as well as the results of treatment of leiomyosarcoma have not changed. Still, the main method of treatment is radical surgical excision of tumors and the effectiveness of adjuvant therapy still remains questionable. The reason behind this can be traced to the insufficient knowledge of the biology of this group of tumors, which in turn results from their rarity and heterogeneity. The biology of leiomyosarcoma differs significantly from the biology of better-known cancers of epithelial origin.

In the literature various survival rates of patients suffering from uterine LMS are mentioned, most of reported rates of 5 years disease specific survival vary from 4 to about 70 percent [21]. The results obtained in the study may seemed to be unexpectedly good, 72 percent of patients survived longer than 2 years after diagnosis. It can be explained by the fact that most of them underwent complete tumor resection.

In several studies the formation of blood vessels has been widely recognized as a critical process in the growth of cancerous tumors. The process of angiogenesis is accompanied by the dynamic development of the lymphatic vessels, called lymphangiogenesis. The role of assessment and the potential usefulness of examining the networks of microvessels in LMS is so far poorly understood. One of the markers of the process of the lymphatic vessels creation is neuropilin 1, present in a mature endothelium of the lymphatic system. It has been shown that the overexpression of this protein in the cancerous tissue is a poor prognostic factor in many malignant tumors [22].

Neuropilin 1 is an important receptor that participates in the regulation of metabolism and the cells migration. In the majority of examined uterine leiomyosarcoma, the positive 
Table 3. Neuropilin 1 expression in the groups of patients depending on their 2-year survival

\begin{tabular}{|l|c|c|c|}
\hline & Survival $\leq \mathbf{2}$ years & Survival > 2 years & $\mathbf{p}^{*}$ \\
\hline N & 14 & 36 & 0.011 \\
\hline No expression or weak expression & $3(21.4 \%)$ & $11(78.6 \%)$ & 0.005 \\
\hline Moderate expression & $23(63.9 \%)$ & $13(36.1 \%)$ & $14(38.9 \%)$ \\
\hline No expression & $0(0.0 \%)$ & $22(61.1 \%)$ & \\
\hline
\end{tabular}

$\mathrm{P}^{*}$ - the significance was calculated with the use of Fisher's test

expression of this protein was discovered. While analyzing the material statistically, the positive correlation between the increased expression of neuropilin 1 and the clinical stage of the disease was noted. The upward trend of this protein expression increase with the development of the disease was also observed. Moreover, a negative correlation between the neuropilin 1 expression and the LMS patients survival time was observed. Similar results were achieved by Lu et all. who analyzed the correlations between neuropilin 1 expression and patients survival and the clinical stage of malignant melanoma [23]. Neuropilin 1 was identified as a prognostic factor in the ovarian cancer in which the positive correlation between its expression and a degree of clinical stage according to FIGO was also observed [24]. The results and the analysis of the available literature, led to conclusion that NRP1 may in the future be a potential prognostic factor in the LMS. It is worth noting that the immunohistochemical assessment of neuropilin 1 expression is a relatively easily available method and can be routinely used as an element of histopathological examination. The presence of NRP 1 overexpression in leiomyosarcoma apart from the prognostic value, can be also used in planning future therapies. Nowadays, the studies focus on the use of antibodies against NRP 1 as components of immunoliposomes containing cytotoxic drug in their interior. They presents the ability ot selectively bind with cells that have NRP 1 on its surface. The aim of creating immunoliposomes containing antibodies against proteins whose expression is high on the surface of cancer cells is enabling the selective uptake by the cancer cells which in turn increases the concentration of the drug in the tumor. Such a procedure on one hand, has a chance to lead to the reduction of cancer therapy toxicity (by reducing the concentration of the drug in other tissues), and on the other hand, to boost its effectiveness by significant increase of cytostatic concentration in the tumor. The studies on the use of immunoliposoms containing in their interior docetaxel and on the surface antibodies against neuropilin 1 was conducted on melanoma and lung cancer models by Manjappa et all. [25]. They observed the selective uptake of liposomes by the cells of both cancers which resulted in the increase of drug cytotoxicity. Moreover, they noted the significant decrease of microvessel density in the tumors that were treated with liposomes. The above observations may suggest that apart from the increase of drug concentration tumors which results in the increase of cytotoxic effect, the use of antibodies against neuropilin 1 limits the angiogenesis process intensification. Such an action can be attributed to the deterioration of the neuropilin function in the cancer cells metabolism as well as in endothelial cells.

Taking into account the results of the studies concerning the neuropilin 1 expression in leiomyosarcoma, it seems reasonable to conduct research on possibilities of therapy with the use of immunoliposoms containing antibodies anti-neuropilin 1, especially with reference to LMS in severe clinical stages of the disease. The introduction of this kind of therapy has a chance to improve treatment results, especially in the cases in which the tumor total surgical resection is impossible. In the conducted research, especially high expression of NRP 1 was observed in tumors of a severe clinical stage which appears to prove the possibility of using such therapies in these cases.

\section{CONCLUSIONS}

On the basis of the study, the following conclusions were made:

1. The expression of NRP1 is associated with clinical advancement and worse prognosis in uterine LMS.

2. Neuropilin 1 can be widely used as a postoperative survival predictor for the patients suffering from uterine LMS.

\section{Details of all funding sources for the work}

The paper was developed using the equipment purchased within the Project "The eqiupment of innovative laboratories doing research on new medicines used in the therapy of civilization and neoplastic diseases" within the Operational Program Development of Eastern Poland 2007-2013, Priority Axis I Modern Economy, Operations I.3 Innovation Promotion. The paper was founded by Medical University of Lublin, grant no. DS 121. 


\section{Conflict of interest}

There are no conflict of interest and financial disclosures from any authors.

\section{REFERENCES}

1. Bodner K, Bodner-Adler B, Kimberger $\mathrm{O}$, et al. Evaluating prognostic parameters in women with uterine leiomyosarcoma. A clinicopathologic study. J Reprod Med. 2003; 48(2): 95-100, indexed in Pubmed: 12621792.

2. Ramondetta L, Bodurka D, Deavers M, et al. Uterine Sarcomas. Gynecologic Cancer. 2006: 125-147, doi: 10.1007/0-387-28796-5_9.

3. Ueda SM, Kapp DS, Cheung MK, et al. Trends in demographic and clinical characteristics in women diagnosed with corpus cancer and their potential impact on the increasing number of deaths. Am J Obstet Gynecol. 2008; 198(2): 218.e1-218.e6, doi: 10.1016/j.ajog.2007.08.075, indexed in Pubmed: 18226630.

4. Wang $Y$, Yao $X, G e J$ J, et al. Can vascular endothelial growth factor and microvessel density be used as prognostic biomarkers for colorectal cancer? A systematic review and meta-analysis. ScientificWorldJournal. 2014; 2014: 102736, doi: 10.1155/2014/102736, indexed in Pubmed: 25143961.

5. Li S, Li Q. Cancer stem cells, lymphangiogenesis, and lymphatic metastasis. Cancer Lett. 2015;357(2):438-447, doi: 10.1016/j.canlet.2014.12.013, indexed in Pubmed: 25497008.

6. He Y, Rajantie I, Pajusola K, et al. Vascular endothelial cell growth factor receptor 3-mediated activation of lymphatic endothelium is crucial for tumor cell entry and spread via lymphatic vessels. Cancer Res. 2005; 65(11): 4739-4746, doi: 10.1158/0008-5472.CAN-04-4576, indexed in Pubmed: 15930292

7. Tezuka K, Onoda N, Takashima T, et al. Prognostic significance of lymphovascular invasion diagnosed by lymphatic endothelium immunostaining in breast cancer patients. Oncol Rep. 2007; 17(5): 997-1003, indexed in Pubmed: 17390035

8. Nasarre P, Gemmill RM, Drabkin HA. The emerging role of class-3 semaphorins and their neuropilin receptors in oncology. Onco Targets Ther. 2014; 7: 1663-1687, doi: 10.2147/OTT.S37744, indexed in Pubmed: 25285016.

9. Shahrabi-Farahani S, Wang L, Zwaans BMM, et al. Neuropilin 1 expression correlates with differentiation status of epidermal cells and cutaneous squamous cell carcinomas. Lab Invest. 2014; 94(7): 752-765, doi: 10.1038/labinvest.2014.66, indexed in Pubmed: 24791743.

10. Handa A, Tokunaga T, Tsuchida T, et al. Neuropilin-2 expression affects the increased vascularization and is a prognostic factor in osteosarcoma. Int J Oncol. 2000; 17(2): 291-295, indexed in Pubmed: 10891538.

11. Fakhari M, Pullirsch D, Abraham $D$, et al. Selective upregulation of vascular endothelial growth factor receptors neuropilin-1 and -2 in human neuroblastoma. Cancer. 2002; 94(1): 258-263, indexed in Pubmed: 11815985.
12. Osada $\mathrm{H}$, Tokunaga $T$, Nishi $M$, et al. Overexpression of the neuropilin 1 (NRP1) gene correlated with poor prognosis in human glioma. Anticancer Res. 2004; 24(2B): 547-552, indexed in Pubmed: 15160992.

13. Kawakami $\mathrm{T}$, Tokunaga $\mathrm{T}$, Hatanaka $\mathrm{H}$, et al. Neuropilin 1 and neuropilin 2 co-expression is significantly correlated with increased vascularity and poor prognosis in nonsmall cell lung carcinoma. Cancer. 2002; 95(10): 2196-2201, doi: 10.1002/cncr.10936, indexed in Pubmed: 12412174.

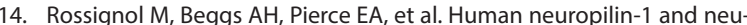
ropilin-2 map to 10p12 and 2q34, respectively. Genomics. 1999; 57(3): 459-460, doi: 10.1006/geno.1999.5790, indexed in Pubmed: 10329017.

15. Wild JRL, Staton CA, Chapple K, et al. Neuropilins: expression and roles in the epithelium. Int J Exp Pathol. 2012; 93(2): 81-103, doi: 10.1111/j.1 365-2613.2012.00810.x, indexed in Pubmed: 22414290.

16. Bednarek W, Mazurek-Kociubowska M, Sobstyl M, et al. Expression of lymphangiogenesis marker neuropilin-1 in different types of ovarian cancer. Ginekol Pol. 2010; 81(3): 176-182, indexed in Pubmed: 20486537.

17. Kärpänen $T$, Heckman $C A$, Keskitalo $S$, et al. Functional interaction of VEGF-C and VEGF-D with neuropilin receptors. FASEB J. 2006; 20(9): 1462-1472, doi: 10.1096/fj.05-5646com, indexed in Pubmed: 16816121.

18. Xu Y, Yuan Li, Mak J, et al. Neuropilin-2 mediates VEGF-C-induced lymphatic sprouting together with VEGFR3. J Cell Biol. 2010; 188(1): 115-130, doi: 10.1083/jcb.200903137, indexed in Pubmed: 20065093.

19. FIGO staging for uterine sarcomas. International Journal of Gynecology \& Obstetrics. 2009; 104(3): 179, doi: 10.1016/j.ijgo.2008.12.009.

20. de Oliveira AT, Pinheiro $C$, Longatto-Filho A, et al. Co-expression of monocarboxylate transporter 1 (MCT1) and its chaperone (CD147) is associated with low survival in patients with gastrointestinal stromal tumors (GISTs). J Bioenerg Biomembr. 2012; 44(1): 171-178, doi: 10.1007/s10863-012-9408-5, indexed in Pubmed: 22281667.

21. Kapp DS, Shin JY, Chan JK. Prognostic factors and survival in 1396 patients with uterine leiomyosarcomas: emphasis on impact of lymphadenectomy and oophorectomy. Cancer. 2008; 112(4): 820-830, doi: 10.1002/cncr.23245, indexed in Pubmed: 18189292.

22. Roth L. The good, the bad and the ugly: a neuropilin-2 story from normal to tumor-associated lymphangiogenesis. Cell Adh Migr. 2008; 2(4): 217-219, indexed in Pubmed: 19262149.

23. Lu J, Cheng Y, Zhang G, et al. Increased expression of neuropilin 1 in melanoma progression and its prognostic significance in patients with melanoma. Mol Med Rep. 2015; 12(2): 2668-2676, doi: 10.3892/mmr.2015.3752, indexed in Pubmed: 25954957.

24. Jiang $H, X i$, Wang F, et al. Increased expression of neuropilin 1 is associated with epithelial ovarian carcinoma. Mol Med Rep. 2015; 12(2): 2114-2120, doi: 10.3892/mmr.2015.3580, indexed in Pubmed: 25845525.

25. Manjappa AS, Goel PN, Gude RP, et al. Anti-neuropilin 1 antibody Fab' fragment conjugated liposomal docetaxel for active targeting of tumours. J Drug Target. 2014; 22(8): 698-711, doi: 10.3109/1061186X.2014.910792, indexed in Pubmed: 24766186. 